\title{
Attosecond science
}

\author{
D. M. Villeneuve \\ Joint Attosecond Science Laboratory, National Research Council of Canada and University of Ottawa, Ottawa, Canada
}

\begin{abstract}
Scientists have been developing sources of light with ever-shorter pulse durations, in order to study motion in systems ranging from a golfer's swing to the motion of atoms within molecules. The shortest pulses produced to date are under 60 attoseconds, i.e. $60 \times 10^{-18} \mathrm{~s}$. One attosecond is to one second as one second is to the age of the universe. For comparison, the classical orbital period of an electron in a hydrogen atom is 150 attoseconds. Attosecond pulses were first produced in 2001. This article describes how attosecond pulses are generated and how they are measured. Some applications of attosecond pulses are described, such as measuring the delay in photoionisation, or observing molecular dissociation dynamics.
\end{abstract}

\section{ARTICLE HISTORY}

Received 25 August 2017

Accepted 14 November

2017

\section{KEYWORDS}

Attosecond; femtosecond; high harmonic generation; photoionisation; transient absorption

\section{Introduction}

Since the flash photography experiments of 'Doc' Edgerton in the 1950's [1], scientists have been developing pulsed light sources with ever-shorter durations. Short pulses of light are used to freeze an instant of time and allow us to observe motion on very fast time scales. While flash photography is used to freeze motion through twodimensional images, short light pulses can be used to study things that cannot be imaged in the conventional sense, such as the motion of molecules. Today, the shortest reported pulse duration is 53 attoseconds [2].

Nanosecond Q-switched lasers led to picosecond mode-locked lasers which led to femtosecond lasers [3]. However, progress hit a wall when the individual optical pulses had a duration on the order of the period of the optical cycle of the laser's carrier frequency, typically a few femtoseconds. This is illustrated in Figure 1. An 800$\mathrm{nm}$ laser wavelength has an optical period of $2.66 \mathrm{fs}$, so it is difficult to create an envelope whose duration is much shorter than this. Mathematically, if the envelope is too short, then the pulse will not propagate in vacuum. The solution is to use a shorter carrier wavelength that can support a shorter envelope. The problem is how to create a short envelope with such a high-frequency carrier.

Figure 2 illustrates different laser schemes based on simplified atomic levels. A 'normal' laser involves transitions between two levels of a neutral atom in which a population inversion has been established. X-ray lasers employ transitions in highly charged ions, whose energy difference is greater than that of neutral atoms. Although
$\mathrm{X}$-ray lasers have been demonstrated in the soft X-ray range, their pulse durations are typically nanoseconds [4].

But is it necessary to use transitions between bound states? What about using continuum states as the upper laser level? This approach would mean that the transition energy is unlimited. But how does one achieve a population inversion in this system? And since the continuum electron must be located near the ion core for recombination to occur, how can this be achieved? The answer to these questions came through an unexpected route, in the process called high harmonic generation, or HHG [5-9].

\section{High harmonic generation}

Consider an atom exposed to an intense laser field. If its intensity is sufficient, the laser field will detach an electron from the atom, as shown in Figure 3. For near-infrared wavelengths, it is usually a valance electron that is removed. The electron now finds itself under the influence of the laser's electric field, and it will be accelerated away from the ion. Within half of an optical cycle, the electron will be driven back toward the ion. This achieves the condition discussed in the previous section and allows the electron to recombine with the parent ion. It will give up its kinetic energy plus binding energy $\left(K+I_{p}\right)$ in the form of a photon.

A purely classical model of electron motion captures the important features of the three-step model [10]. Consider a one-dimensional model with the atom at the origin. If the electron is freed from the atom at time $t^{\prime}$ and 

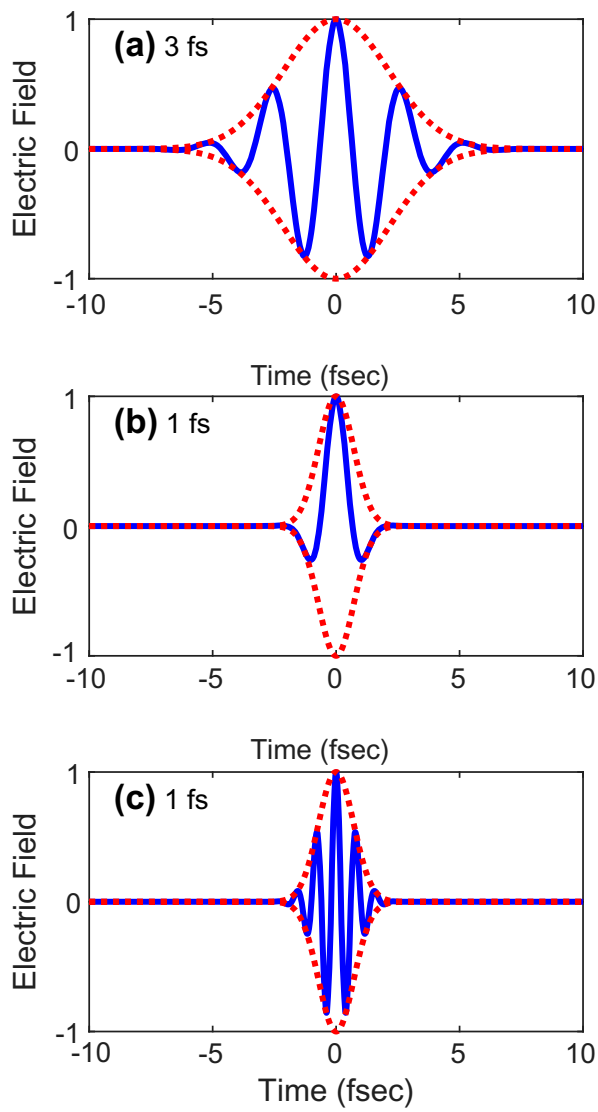

Figure 1. Illustration of why creating ever-shorter laser pulse durations requires shorter wavelengths. (a) A 3-fs duration laser pulse. (Blue) Electric field of laser with an 800-nm carrier wavelength, (red dashed) envelope giving a 3-fs FWHM intensity duration. Such pulses can be achieved today with Ti:Sa lasers. (b) Same 800-nm laser wavelength with an envelope of 1-fs duration. Since the electric field now has a DC term, such a pulse cannot propagate. (c) Same 1-fs envelope but with a shorter wavelength carrier. This pulse can now propagate, but is in the ultraviolet portion of the spectrum.

the effect of the Coulomb potential is ignored, then the electron's trajectory is due only to the oscillating field:

$$
\begin{aligned}
a(t) & =\frac{e}{m} E(t)=\frac{e E_{0}}{m} \cos \omega t, \\
v(t) & =\frac{e E_{0}}{m \omega}\left(\sin \omega t-\sin \omega t^{\prime}\right), \\
x(t) & =-\frac{e E_{0}}{m \omega^{2}}\left(\cos \omega t-\cos \omega t^{\prime}+\left(t-t^{\prime}\right) \sin \omega t^{\prime}\right) .
\end{aligned}
$$

Here, $a, v$ and $x$ refer to the electron's acceleration, velocity and position, respectively. $E$ is the external oscillating electric field, and $\omega$ is the angular frequency of the field. We have applied the initial conditions $x\left(t^{\prime}\right)=v\left(t^{\prime}\right)=0$. There are certain trajectories that return to the parent ion at later times, i.e. have solutions to $x(t)=0$.

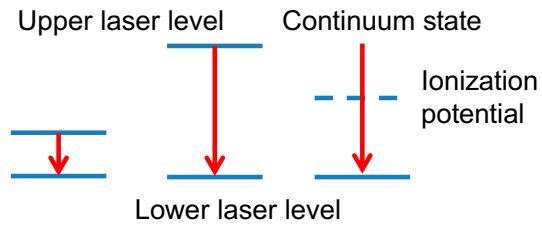
(a) Visible
(b) X-ray
(c) High
light laser
laser
harmonic
generation

Figure 2. Simplified diagrams of atom transitions for lasing. (a) A 2-level system in which the lasing transition is between two bound states. (b) In a highly charged ion, the energy between states is significantly increased compared with a neutral atom, leading to emission in the XUV. This is the scheme used by X-ray lasers. (c) If the upper state is in the continuum, then there is no limit to the photon energy of the transition. The problem is to make the electron recombine with the atom. This is solved in high harmonic generation, in which the electron recollides with the parent atom.

When the electron returns to the parent ion, three things can occur. The electron can elastically scatter from the ion, the electron can inelastically scatter from the ion and the electron can photorecombine with the ion. The latter process is very unlikely, but nevertheless is responsible for the field of attosecond science. During photorecombination, the kinetic energy that the electron has picked up from the laser field is converted into a single photon that is emitted by the atom.

Depending on the exact time that the electron is removed from the atom, its classical trajectory will return it to the ion core at slightly different times and with different kinetic energies. The maximum kinetic energy that the electron can achieve upon recollision depends only on the instantaneous laser intensity $I$ and the laser wavelength $\lambda$, as follows: $K_{\max }=3.17 U_{p}$, where the ponderomotive potential is given by $U_{p}=\left(e E_{0}\right)^{2} /\left(4 m \omega_{0}^{2}\right), E_{0}$ is the peak electric field and $\omega_{0}$ is the angular frequency of the laser field [10]. Including the binding energy of the electron $I_{p}$, the maximum photon energy possible by recombination is $\hbar \Omega=3.17 U_{p}+I_{p}$. For a typical titanium sapphire femtosecond driving laser $(\lambda=800 \mathrm{~nm})$, photon energies in the range of 10 to $150 \mathrm{eV}$ are possible.

The above was a semi-classical description of HHG. The three-step model can be reformulated in a quantum mechanical picture, as shown in Figure 4. The initial electron wavefunction is bound to the ionic core by the Coulomb potential. The instantaneous electric field of the laser causes the Coulomb barrier to be suppressed, allowing some of the bound wavefunction to escape the potential. The free part of the wavefunction will be accelerated back to the core by the field and can interfere with the remaining part of the bound wavefunction. Since this is a single wavefunction representing the electron, it can interfere with itself. The expectation value of the 


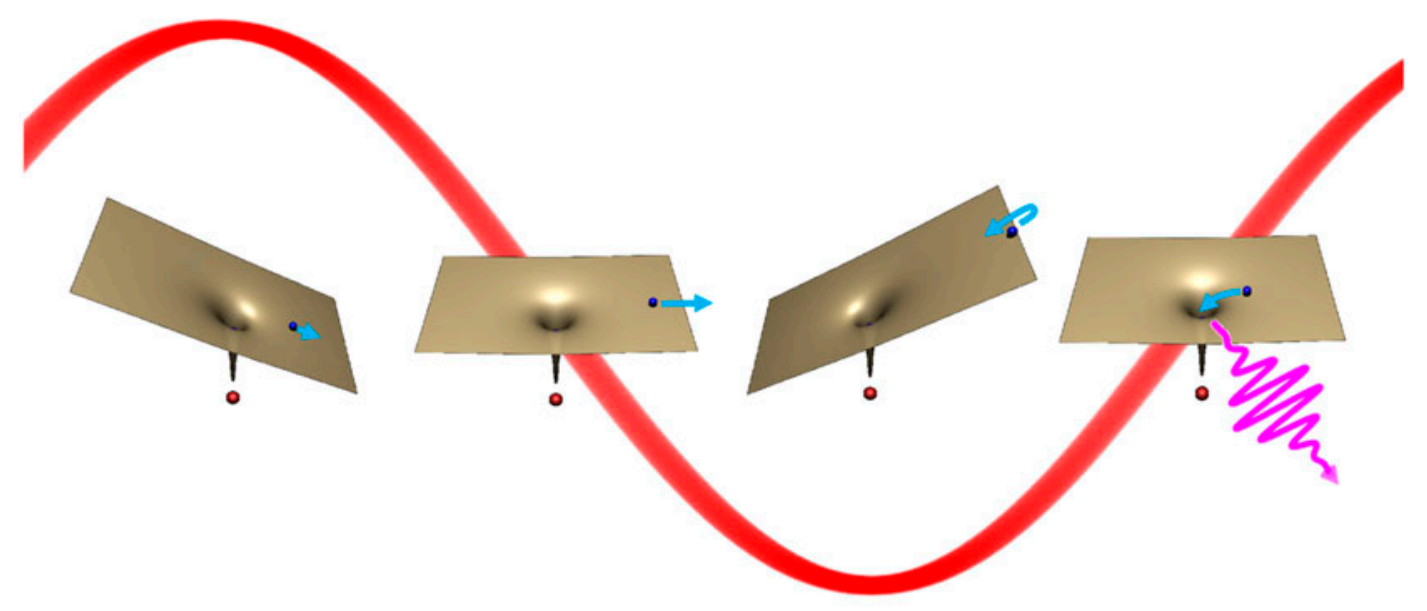

Figure 3. Illustration of three-step model of HHG. In the first step (left), the intense laser field (shown in red) will detach an electron from the atom near the peak of the field oscillations. In the second step (middle two images), the electron is pushed away from the atom by the laser field and then will be pushed back toward the atom when the field changes direction. In the third step (right), the electron can photo-recombine with the parent ion, converting its kinetic energy into a photon. This process is coherent, since the emitted electromagnetic field depends only on the laser field cycle.

(a)

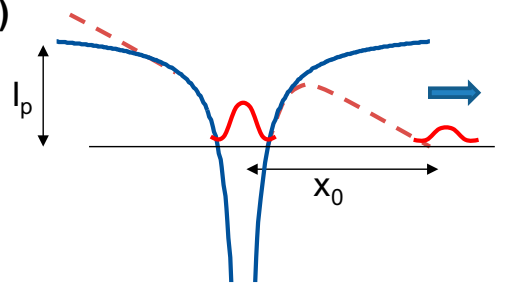

(b)

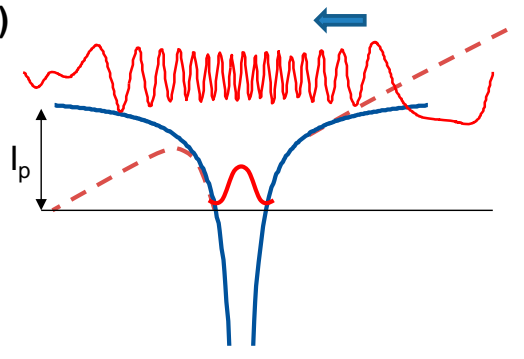

$\psi=\psi_{0}+\psi_{\epsilon}$, and include the time dependence of each, then the expectation value of the position is

$$
\begin{aligned}
\langle x\rangle & =\langle\psi|x| \psi\rangle \\
& =\left\langle\psi_{0} e^{i I_{p} t / \hbar}|x| \psi_{\epsilon} e^{-i \epsilon t / \hbar}\right\rangle \\
& =\left\langle\psi_{0}|x| \psi_{\epsilon}\right\rangle e^{-i\left(\epsilon+I_{p}\right) t / \hbar} .
\end{aligned}
$$

The three-step model can be described quantum mechanically by the time-reversed S-matrix method [11], also called the strong field approximation (SFA):

$$
\begin{aligned}
\boldsymbol{d}(t)=- & i \int d \boldsymbol{k} \int_{0}^{t} d t^{\prime}\langle\psi|\boldsymbol{r}| \boldsymbol{k}+\boldsymbol{A}(t)\rangle e^{-i S} \boldsymbol{E}\left(t^{\prime}\right) \\
& \cdot\left\langle\boldsymbol{k}+\boldsymbol{A}\left(t^{\prime}\right)|\boldsymbol{r}| \psi\right\rangle .
\end{aligned}
$$

Here, $\boldsymbol{d}$ is the dipole response of the atom, $\boldsymbol{k}$ is the canonical momentum, $\boldsymbol{A}$ is the vector potential of the laser field, $\psi$ is the single-electron wavefunction describing the bound electron in the atom and $S=\int_{t^{\prime}}^{t} d t^{\prime \prime}(\boldsymbol{k}+$ $\left.\boldsymbol{A}\left(t^{\prime \prime}\right)\right)^{2} / 2+I_{p}\left(t-t^{\prime}\right)$ is the classical action, with $I_{p}$ being the ionisation potential. Reading this expression from right to left, ionisation occurs at time $t^{\prime}$, the electron accumulates a Volkov phase in the continuum described by the classical action $S$ and recombination back to $\psi$ occurs at time $t$.

The radiated electromagnetic spectrum [12] is the square of the Fourier transform of the second derivative of $d(t)$, namely $S(\Omega)=\Omega^{4}\left|\int d(t) e^{-i \Omega t} d t\right|^{2}$.

This is the single-atom part of the process of HHG. Because the process begins and ends with the atom in the state described by $\psi$, the phase of $\psi$ cancels out. This means that the process is coherent, independent of the

position $\langle x\rangle$ will oscillate at a frequency given by the energy difference of the two parts of the wavefunction. If we write the wavefunction as the sum of the two parts, 

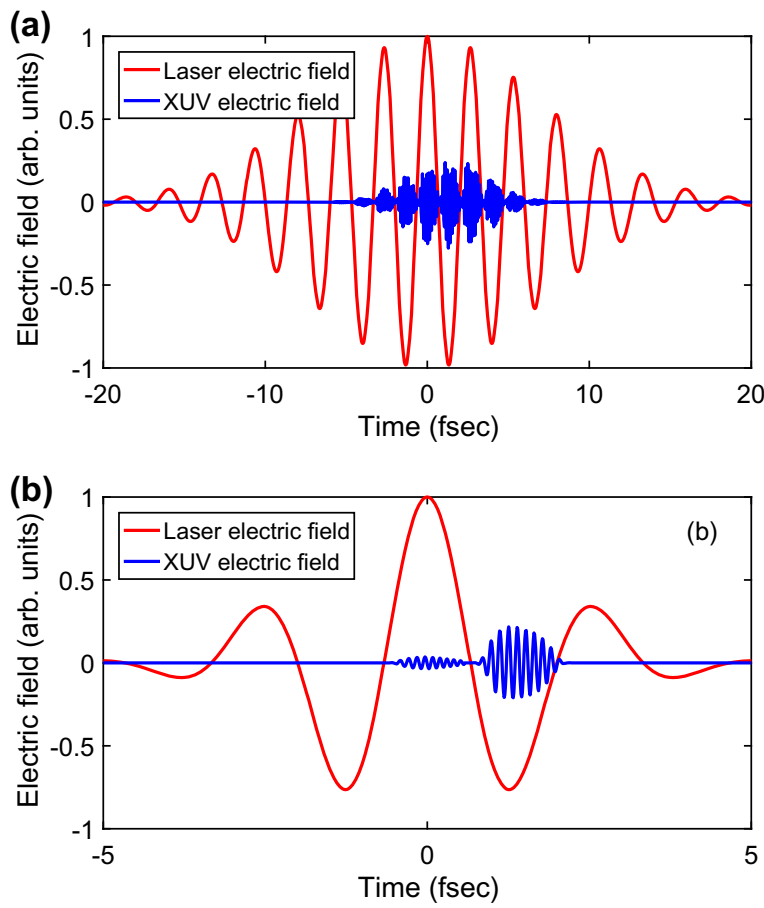

Figure 5. Relationship between the driving infrared laser field and the attosecond pulses it produces. (a) A multicycle infrared pulse (red) creates a series of attosecond pulses (blue). Due to the periodicity in time of the pulse train at twice the frequency of the infrared field, the power spectrum will contain a series of odd harmonics of the laser frequency. This is why the process is called high harmonic generation. (b) A few-cycle laser pulse is now employed to produce a single attosecond pulse. Because the infrared pulse is so short, it is important to control the phase of the infrared carrier within the pulse envelope. This is called the carrier-envelope phase (CEP). Depending on the value of the CEP, a few-cycle pulse can produce either one or two attosecond pulses. Control of the CEP is an important requirement for the generation of isolated attosecond pulses.

phase of each atom. Recombination to excited states is also possible, but does not result in coherent emission. Thus HHG is very sensitive to the wavefunction of the atom and ignores other processes that do not end at the initial state [13].

The quantum model includes all possible classical trajectories at the same time, so different ionisation times result in different return times and kinetic energies. This results in an electronic wave packet whose kinetic energy at the origin first increases, then decreases. This results in an emitted pulse that chirps up in frequency, then down. This is called 'attochirp'.

We use the strong field approximation (Equation (7)) to calculate the electric field that is emitted by an atom exposed to an infrared laser pulse, and shown in Figure 5.

The three-step model describes what happens during a single optical cycle. The whole process repeats every half optical cycle, resulting in the emission of a train of attosecond pulses. Each attosecond pulse chirps up to the maximum frequency given by the $3.17 U_{p}+I_{p}$ cutoff limit, then down again. By the Fourier repetition theorem, any waveform that repeats with a period $T$ will have a spectrum whose envelope is the Fourier transform of the single waveform, multiplied by a series of delta functions with separation of $1 / T$. That is, if the waveform of a single attosecond pulse is $f(t)$ and whose Fourier transform is $F(\omega)$ and if $f(t)$ is repeated with period $T$, then the spectrum of a pulse train is

$$
F^{\prime}(\omega)=\frac{2 \pi}{T} F(\omega) \sum_{n} \delta\left(\omega-n \frac{2 \pi}{T}\right) .
$$

So we would expect the spectrum of the attosecond pulse train to be a series of delta functions separated by $2 \omega_{0}$. Because of the alternating sign of each attosecond pulse (due to the alternating left and right directions of the laser field), this will be a series of odd harmonics of the driving laser field. This explains the name 'high harmonic generation'.

Figure 6 shows what a typical source of high harmonic radiation looks like. An intense femtosecond laser pulse is focused into a gas jet or a gas cell or a capillary where it ionises the gas, leading to emission of the attosecond pulse train in the same direction as the laser pulse. The attosecond pulse train then propagates into a different vacuum chamber where it is used to perform an experiment. In the figure, the HHG emission is measured using an XUV spectrometer.

If one wants a single attosecond pulse, then it is usually necessary to have a driving laser pulse of very short duration. This will limit the three-step model to a single half optical cycle, resulting in a single attosecond pulse as illustrated in Figure 5(b). There are other approaches to creating isolated attosecond pulses, such as polarisation gating [14], ionisation gating [15] or photonic streaking [16]. Remarkably, the attosecond pulse that is created has a consistent shape from shot to shot, and even the shape of the electric waveform under the envelope is controlled (carrier-envelope phase) [17].

As described above, the highest emitted photon energy scales as $3.17 U_{p}$, or as $I \lambda^{2}$. It would seem that the cutoff energy can be scaled arbitrarily high simply by increasing the laser intensity $I$. This does not work. As the laser intensity increases, more of the electron wavefunction is removed from the atom. When all of the wavefunction is removed (complete ionisation), there is no longer any bound state wavefunction to interfere with the continuum wavefunction, and emission will cease. (This effect is exploited in ionisation gating [15].) Peak laser intensity for HHG is therefore practically limited to $I<10^{15} \mathrm{~W} / \mathrm{cm}^{2}$. 


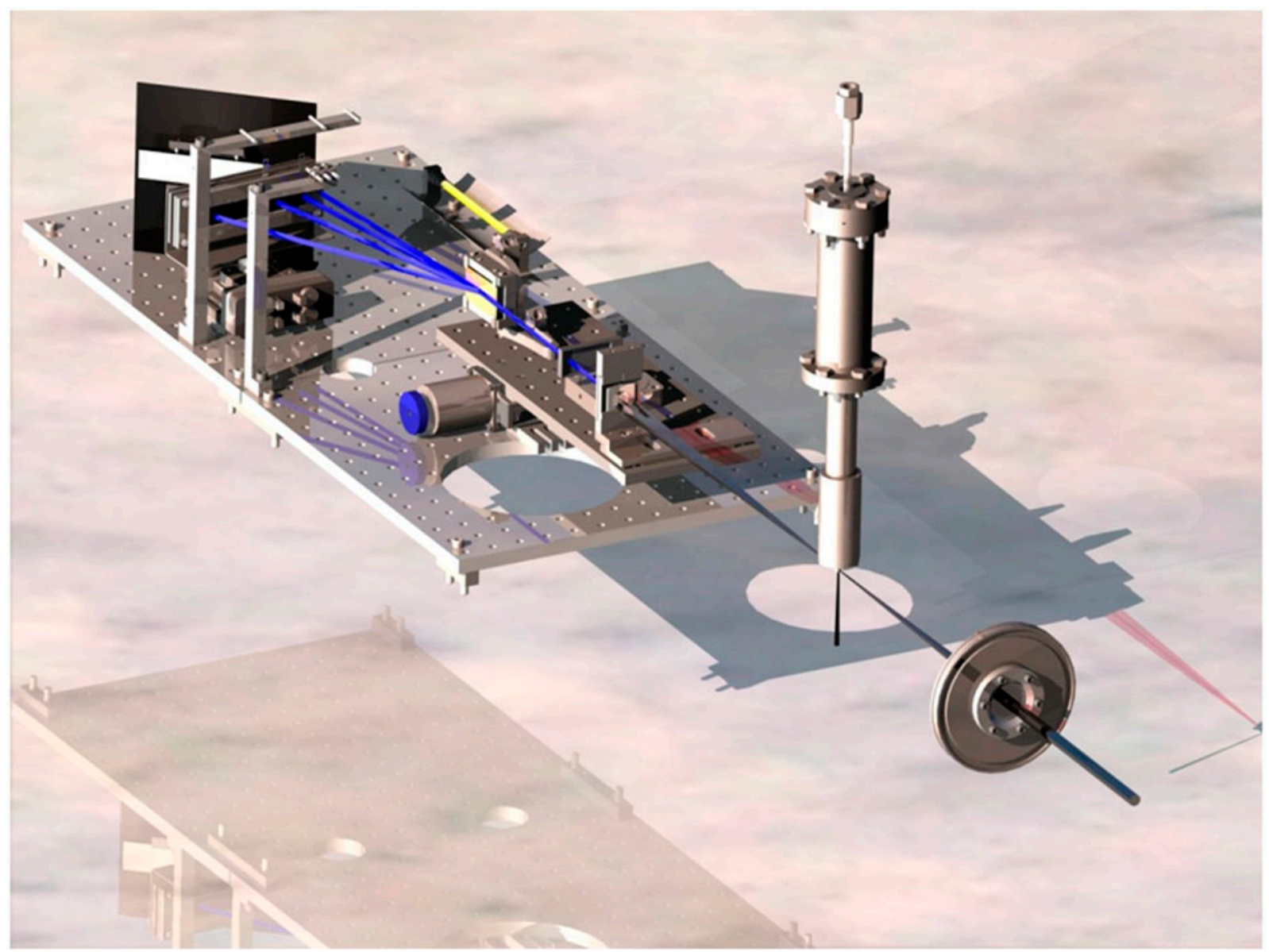

Figure 6. An illustration of a typical high harmonic generation experimental setup. An intense femtosecond laser pulse enters a vacuum chamber (not shown) from the right and is focused into a gas jet. The laser pulse is sufficiently intense to ionise the target gas, leading the phase-matched emission of a train of attosecond pulses in the forward direction. The attosecond pulse train propagates into a second vacuum chamber which provides differential pumping to improve the vacuum. Here, the second chamber contains an XUV spectrometer which records the HHG spectrum. In other setups, the second chamber might contain a second gas jet which is photoionised by the attosecond pulse train.

The $I \lambda^{2}$ scaling suggests another approach to increasing the cutoff energy: increasing the laser wavelength $\lambda$ [18]. The $800 \mathrm{~nm}$ wavelength of Ti:Sapphire laser systems is very convenient, but techniques are available to create few-cycle optical pulses in the near- and mid-IR. For example, the optical parametric amplifier (OPA) [19] can create signal and idler wavelengths whose frequency sum equals the input laser frequency, giving output wavelengths in the range of $1-4 \mu \mathrm{m}$. Optical parametric chirped pulse amplification (OPCPA) [20] utilises nonlinear optical crystals to amplify an infrared pulse. Frequency-domain optical parametric amplification (FOPA) [21] is another approach that does not require the pulse to be chirped.

A downside of using longer-wavelength laser sources for HHG is the reduced efficiency of the HHG process. This is because the wavefunction of the continuum electron spreads in three dimensions, and with the lower optical frequency of longer wavelength sources, the wave- function has more opportunity to spread. The spreading of the continuum wavefunction is shown in Figure 7; the continuum wavefunction has spread to 100 Bohr in radius as it returns to the ion core. Since the spreading increases with increasing wavelength, this means that the portion of the continuum wavefunction that is able to interfere with that part of the electron's wavefunction that is still bound to the atom becomes smaller. This fact was established theoretically [22] and experimentally [23], showing that the HHG efficiency scales approximately as $\lambda^{-6}$. However, there are some techniques that can make up for the smaller single-atom efficiency, for example by increasing the gas pressure [24,25].

The explanation of HHG so far has been limited to a single atom. Surprisingly, this process can be phasematched over a volume of atoms [26,27]. The emitted attosecond pulse only depends on the local intensity and phase of the driving laser. Figure 8 illustrates that each atom will emit a coherent burst of attosecond pulses 


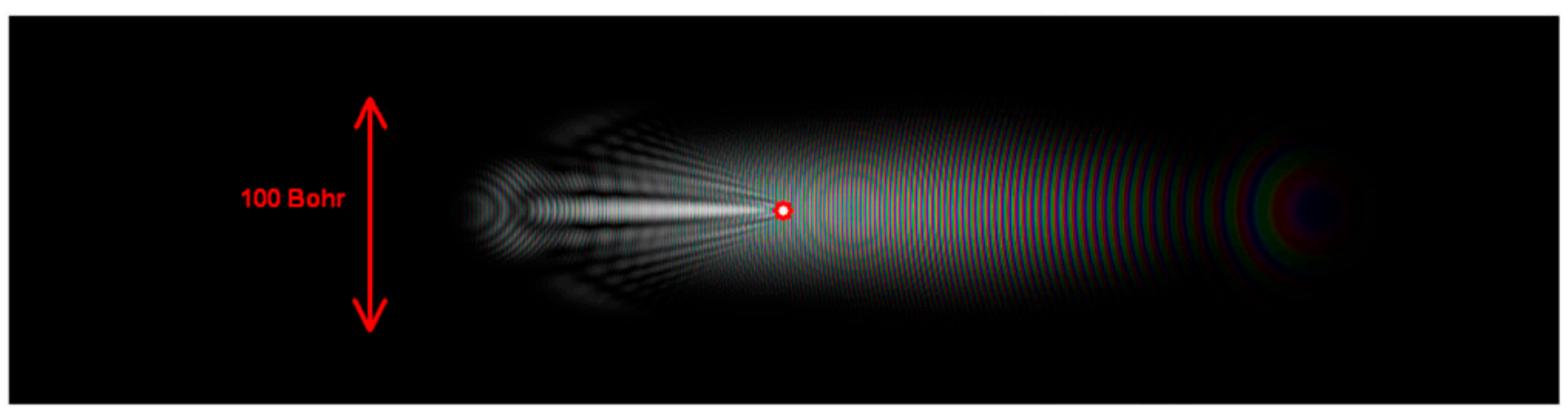

Figure 7. Illustration of the continuum wavefunction as it returns to the ion core. A time-dependent Schrodinger calculation of a hydrogen atom in an intense laser field is shown in two dimensions. The density of the wavefunction is depicted by the brightness, and the phase of the wavefunction by the colour. The atom is marked by the red circle. The laser field initially removed the electron's wavefunction from the core by ionisation. The wavefunction moved to the right. That part of the wavefunction that lies to the left of the circular structure on the right will return to the core, whereas that part that lies to right will never return. The structures on the left are due to the beginning of ionisation in the left direction by the next half optical cycle. The fringes show the phase of the complex wavefunction, and the separation between fringes denotes the de Broglie wavelength and hence the kinetic energy. The kinetic energy of the recolliding electron wave packet increases with time, leading to a chirp in the attosecond pulse frequency.

Figure 8. The single-atom picture shown in Figure 4 is repeated for each atom in the focal volume of the laser. Each atom emits a train of attosecond pulses. Remarkably, the emission from the atoms is locked to the optical phase of the driving laser field, resulting in phase-matched emission. The emission is collimated in the forward direction, usually with a smaller divergence than the driving laser.

depending on its location within the laser focus. The coherent addition of the individual electric fields will result in a forward-propagating train of pulses that usually has a smaller beam divergence than that of the driving laser. Since the gas sample is ionised by the driving laser, other processes can also produce emission, for example recombination radiation; however, these processes are incoherent and result in emission into $4 \pi$, whereas the HHG is phase-matched in the forward direction. The HHG emission will dominate over the incoherent processes.

\section{Measurement of pulse duration}

The coherence of the HHG process means that the attosecond pulse train is perfectly synchronised with the optical cycles of the driving laser pulse. This allows the driving laser pulse to be used as a probe of the attosecond pulse train and is the basis for most techniques used to measure the duration of the attosecond pulses.
The first technique [29] used to determine the duration and shape of a train of attosecond pulses was called RABBIT (original spelling was RABITT: Reconstruction of attosecond harmonic beating by interference of twophoton transitions). In RABBIT, the attosecond pulse train is imaged from the generating gas source into a second, detection, gas, as seen in Figure 9(a). The attosecond pulses photoionise the detection gas, and produce a series of photoelectron peaks whose kinetic energy is given by $K_{q}=q \hbar \omega_{0}-I_{p}$, where $q$ is an odd integer. The photoelectrons are energy resolved by a time-of-flight electron spectrometer.

At the same time, part of the infrared laser pulse that generated the attosecond pulses is also focused into the detection gas. The infrared pulse can be delayed with respect to the attosecond pulses, for example using a split mirror. (The timing jitter between the two pulses must be shorter than the attosecond pulse duration, so inline interferometers are preferred over Michelson interferometers.) The combination of the infrared and attosecond 
(a) Ex-situ measurements

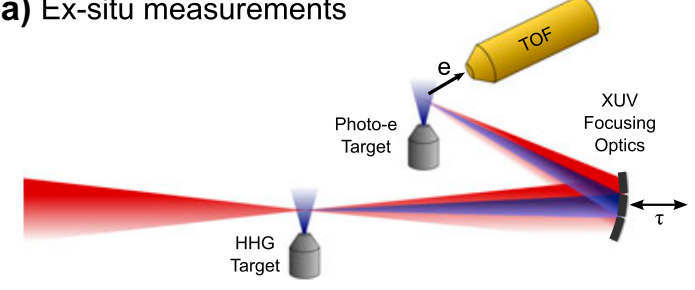

(b)

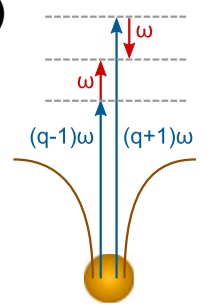

(c)

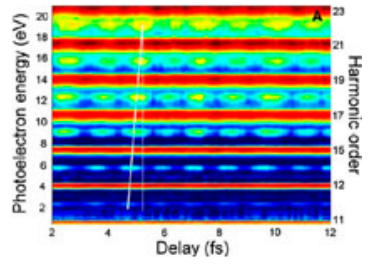

Figure 9. Measurement of an attosecond pulse train using the RABBIT technique. (a) The driving laser pulse is focused into the first gas jet to generate the HHG emission (blue). The emission is focused into a second gas jet which is photoionised. The spectrum of the photoelectron kinetic energy is recorded by a time-of-flight electron spectrometer. Part of the driving laser field (red) is also focused into the second gas jets and serves to modulate the kinetic energy of the photoelectrons, depending on the exact sub-cycle delay. (b) The attosecond pulse train generates a series of photoelectron peaks whose kinetic energy corresponds to the photon energy of harmonic $q$ minus the binding energy of the atom. The dressing laser field adds or subtracts a single photon, resulting in additional photoelectron energies between the harmonic peaks. By varying the delay between the two fields, a RABBIT trace results. The delay at which each sideband maximises determines the relative phase between the adjacent harmonics. This measurement gives the harmonic phase of the HHG emission, from which an average attosecond pulse shape can be determined. (Figure is derived from Ref. [28] with permission from Nature Publishing Group.)

pulses during the ionisation process leads to the creation of sidebands between the photoelectron peaks directly generated by the harmonics. As the delay between the two pulses is varied, the amplitude of the sidebands is modulated at twice the infrared laser frequency, as shown in Figure 9(b). Sideband $n$, where $n$ is an even integer, is modulated by the time delay $\tau$ as

$$
S_{n}(\tau) \propto \cos \left(2 \omega_{0} \tau+\phi_{n-1}-\phi_{n+1}+\Delta \phi_{\text {atomic }}\right) .
$$

Here, $\Delta \phi_{\text {atomic }}$ represents the phase difference due to the atomic recombination phase of the detection gas. The time delay at which each sideband $n$ maximises determines the phase difference between the harmonic orders above and below the sideband. By assuming the phase of one harmonic order, one can step through the RABBIT spectrum and determine the phases between all harmonic orders.

From the determination of the phases $\phi_{q}$ for each harmonic order $q$, together with the amplitude of each harmonic $A_{q}$, it is possible to reconstruct the shape of the average pulse in the attosecond pulse train [30]:

$$
I(t)=\left|\sum_{q} A_{q} e^{-i \omega_{q} t+i \phi_{q}}\right|^{2} .
$$

Here $\phi_{q}$ is called the harmonic phase. It is the phase of harmonic $q$ averaged over the entire pulse train. It is related to, but not the same as, the attosecond chirp. The attosecond chirp refers to the temporal chirp of a single attosecond pulse within the train. Since the frequency of an attosecond pulse increases with time, the chirp can be characterised by a linear chirp, i.e. a quadratric phase. Metal filters have been used to compress attosecond pulses by compensating for the attosecond chirp [31].

To measure the temporal shape of a single-isolated attosecond pulse, the attosecond streak camera was developed [32]. This is in analogy to electro-optic streak cameras, in which a pulse of light is converted into an electron pulse by a photocathode, and the electrons are deflected by plates upon which a ramped voltage is applied. The electrons are detected on a phosphor screen, with the time axis along the deflection direction. For the attosecond streak camera, no voltage can be changed quickly enough, so the infrared laser field itself is used to deflect the electrons that are photoionised from an atom by the attosecond pulse. The infrared electric field $E(t)$ determines the motion of the electron through its vector potential, $E(t)=d A(t) / d t$. As $t \rightarrow \infty$, the velocity of an electron that is detached from the atom with initial velocity $v_{o}$ is $v=v_{0}+\frac{e}{m_{e}} A\left(t_{i}\right)$, where $t_{i}$ is the time at which the electron is detached. This expression provides a mapping between time of ionisation and final velocity of the electron.

A more complete expression for the distribution of electron velocities is [32]

$$
a_{v}(T)=-i \int_{-\infty}^{T} d t d_{p(t)} E_{x}(t) e^{-i \int_{t}^{T} d t^{\prime}\left(p\left(t^{\prime}\right)^{2} / 2+I_{p}\right)}
$$

where $p(t)=v+A(t)$ is the instantaneous kinetic momentum, $d_{p(t)}$ is the dipole transition matrix element from the ground state to the continuum with momentum $p(t)$ and $E_{x}(t)$ is the electric field of the attosecond pulse to be measured.

The RABBIT method can be combined with streaking in a method called FROG-CRAB, that is able to reconstruct the shape of both the attosecond pulse and the driving laser pulse shape [33]. The reconstruction is illustrated in Figure 10. 


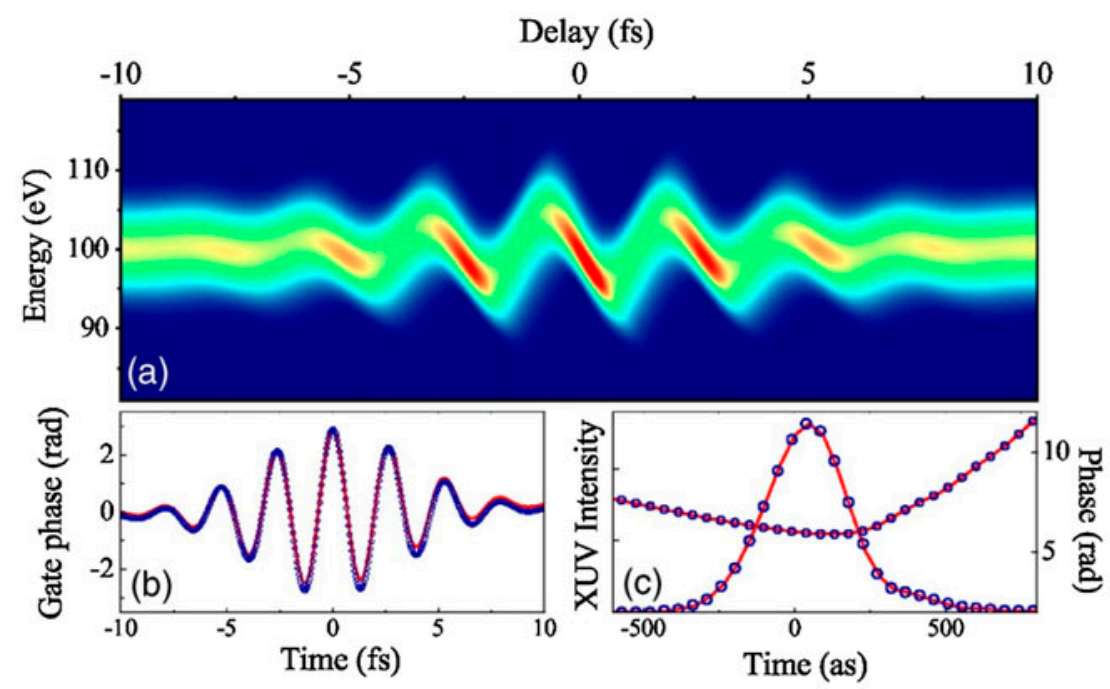

Figure 10. FROG-CRAB measurement of a single attosecond pulse. The upper trace is the photoelectron kinetic energy spectrum vs. delay between the two fields. The infrared dressing field modifies the kinetic energy of electrons produced by the attosecond pulse. The algorithm is able to determine both the shape of the full infrared laser pulse (b) and the temporal amplitude and phase of the attosecond pulse (c). (Figure is derived from Ref. [33] with permission of the American Physical Society.)

The attosecond pulse characterisation techniques discussed so far can be classified as 'ex situ' measurements, since the measurement takes place in a second gas jet. This has the advantage that the measurement often takes place in the same medium in which the experiment is to take place. There is a second type of attosecond measurement called 'in situ', in which the measurement takes place in the medium in which the attosecond pulses themselves are generated. This approach is experimentally easier than ex situ techniques, but has the disadvantage that the actual electromagnetic field is not measured [34].

Figure 11 illustrates one in situ approach [35]. The HHG spectrum is recorded on an XUV spectrometer, revealing the odd harmonics. A weak ( $0.1 \%$ intensity) second harmonic of the driving laser frequency is generated with a second harmonic crystal in the beam. The delay between the two frequencies is adjusted by a glass plate. The second harmonic field breaks the left-right symmetry of the electron trajectories, leading to the emission of even harmonic orders. This looks very much like the RABBIT trace shown previously, and the analysis is similar. This allows the harmonic amplitude and phase to be determined.

A variant of the in situ measurement is the petahertz optical oscilloscope [36], shown in Figure 12. In this measurement, the second harmonic field enters the gas jet at an angle, leading to a phase shift between $\omega$ and $2 \omega$ fields that varies in the vertical direction in the laser focus. The spatially dependent phase shift causes the phase of the harmonic emission to vary in a similar way, leading to a steering of the HHG emission in the vertical direction.

\section{(a) In-situ measurements}

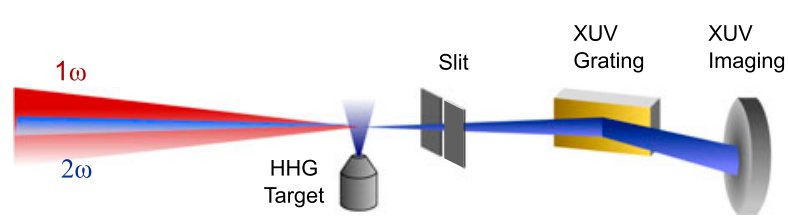

(b)

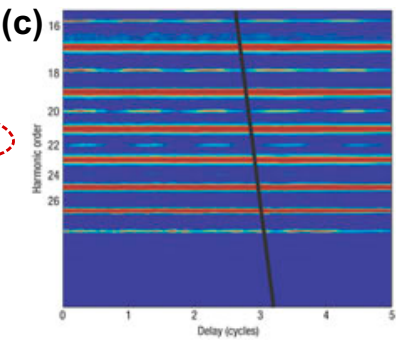

Figure 11. In situ measurement of the harmonic phase of an attosecond pulse train. (a) The HHG spectrum is recorded with an XUV spectrometer. A weak second harmonic of the driving laser frequency slightly perturbs the electron trajectories, causing an imbalance in the left-right symmetry (b) and resulting in the emission of even-order harmonics seen in the spectrum (c). The spectrum is recorded as a function of time delay between the $1 \omega$ and $2 \omega$ fields. Like the RABBIT technique, the delays at which the even harmonics are maximised are measure of the harmonic phase. (Figure is derived from Ref. [28] with permission of the Nature Publishing Group.)

By recording the vertical deviation of the emission as a function of delay, the electric field of the laser pulse can be reconstructed. If the main pulse creates a single attosecond pulse, its spectral amplitude and phase can also be reconstructed. This technique can either be used to characterise the attosecond pulse [37], or to characterise the laser pulse shape (petahertz optical oscilloscope) [36]. 


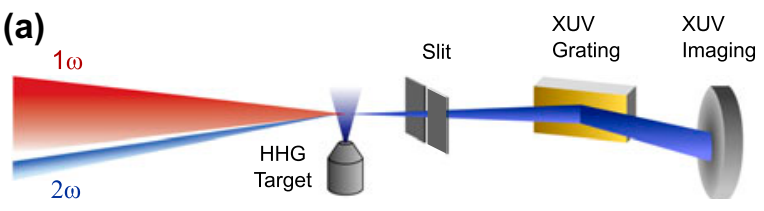

(b)

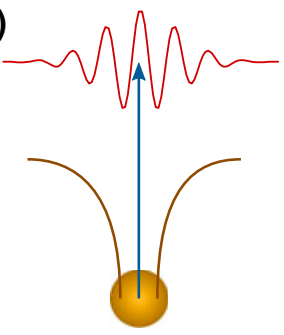

(c)

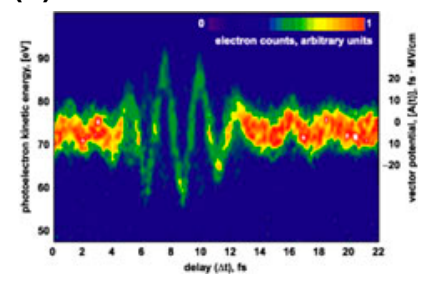

(d)

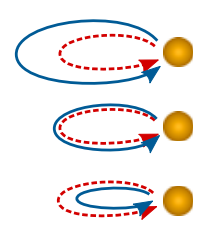

(e)

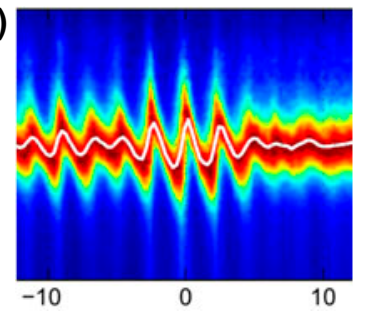

Figure 12. Petahertz optical oscilloscope. A weak second harmonic of the driving laser is focused into the HHG gas jet at an angle to the main beam, causing a slight tilt to the phase front of the emitted harmonics. This causes the HHG emission to be deflected in the vertical direction. The deflection as a function of delay between the two pulses can be used to characterise the attosecond pulse duration and the laser pulse shape. (Figure is derived from Ref. [28] with permission of Nature Publishing Group.)

Finally, we describe the 'photonic streaking' approach, which is both a method to characterise attosecond pulses or pulse trains and in addition can be used to select a single attosecond pulse from a train [16,38]. It is also useful to visually check that the carrier-envelope phase of the laser system is controlled.

Photonic streaking, or the 'attosecond lighthouse', is easy to set up, as illustrated in Figure 13. It requires a short driving pulse with a broad bandwidth. A pair of thin prisms is inserted in the laser beam before the focusing optics. One of the prisms is rotated to create chromatic aberration in the focus. This causes the wavefront to rotate as it passes through the focus. Each attosecond pulse follows the direction of the phase front of the part of the waveform that creates it. This rotation causes each attosecond pulse in the train to be emitted in slightly different directions. It has been shown that the individual pulses in the attosecond lighthouse preserve the temporal characteristics of more usual attosecond sources [39].

\section{Photoionisation delay}

We now turn to applications of attosecond science after having demonstrated the tools. Having a source of attosecond pulses permits us to look at processes that occur on the attosecond time scale. One example is the photoionisation of atoms: how long does it take for the electron to be removed, and does it take longer for deeperbound electrons to be removed? One difficulty with any attosecond measurement is the lack of a time-zero. This means that most attosecond experiments must be made relative to another channel.

Since the attosecond pulse that is used to photoionise an atom necessarily has a broad spectral bandwidth, one expects that the photoelectron wave packet that is produced will be chirped in time. This is because the transition dipole matrix element of the transition from bound to continuum states $d_{\epsilon}=\left\langle\phi_{\epsilon}|r| \psi\right\rangle$ has an energydependent phase shift $\eta(\epsilon)$. A phase shift that is linear with energy is equivalent to a time shift of the electron wave packet relative to the attosecond electromagnetic pulse. This is called the Wigner time delay [40], also called

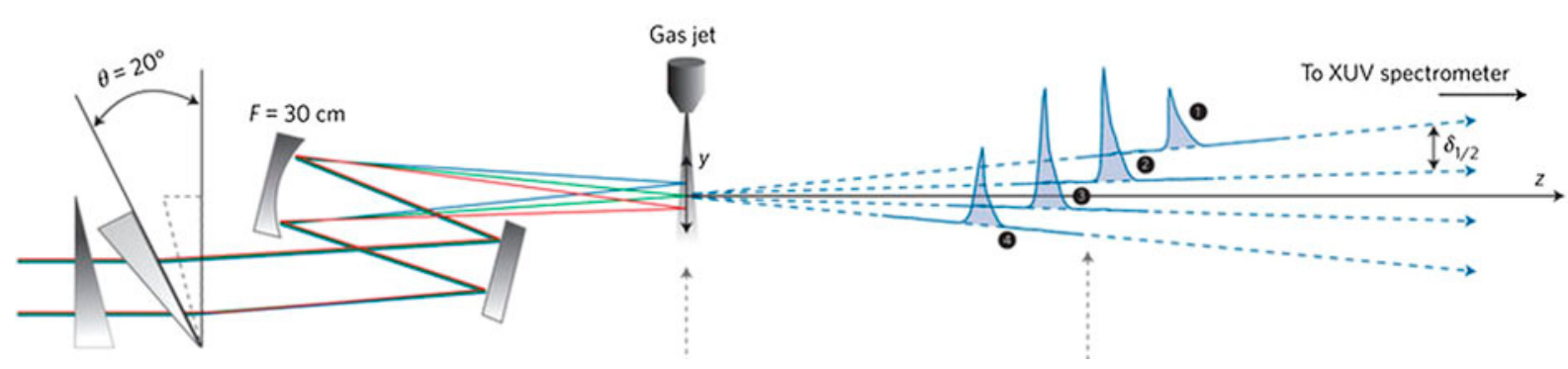

Figure 13. Illustration of photonic streaking of an attosecond pulse train, also called the attosecond lighthouse. Chromatic aberration is applied to the incoming infrared laser field by a twisted pair of thin wedges. The aberration causes the wavefronts in the focus to rotate during the pulse. Since the attosecond pulses are phase-matched perpendicular to the phase front of the driving laser, each attosecond pulse will propagate in a slightly different direction. A single attosecond pulse may be selected by inserting an aperture after the gas jet. (Figure is derived from Ref. [16] with permission of Nature Publishing Group.) 

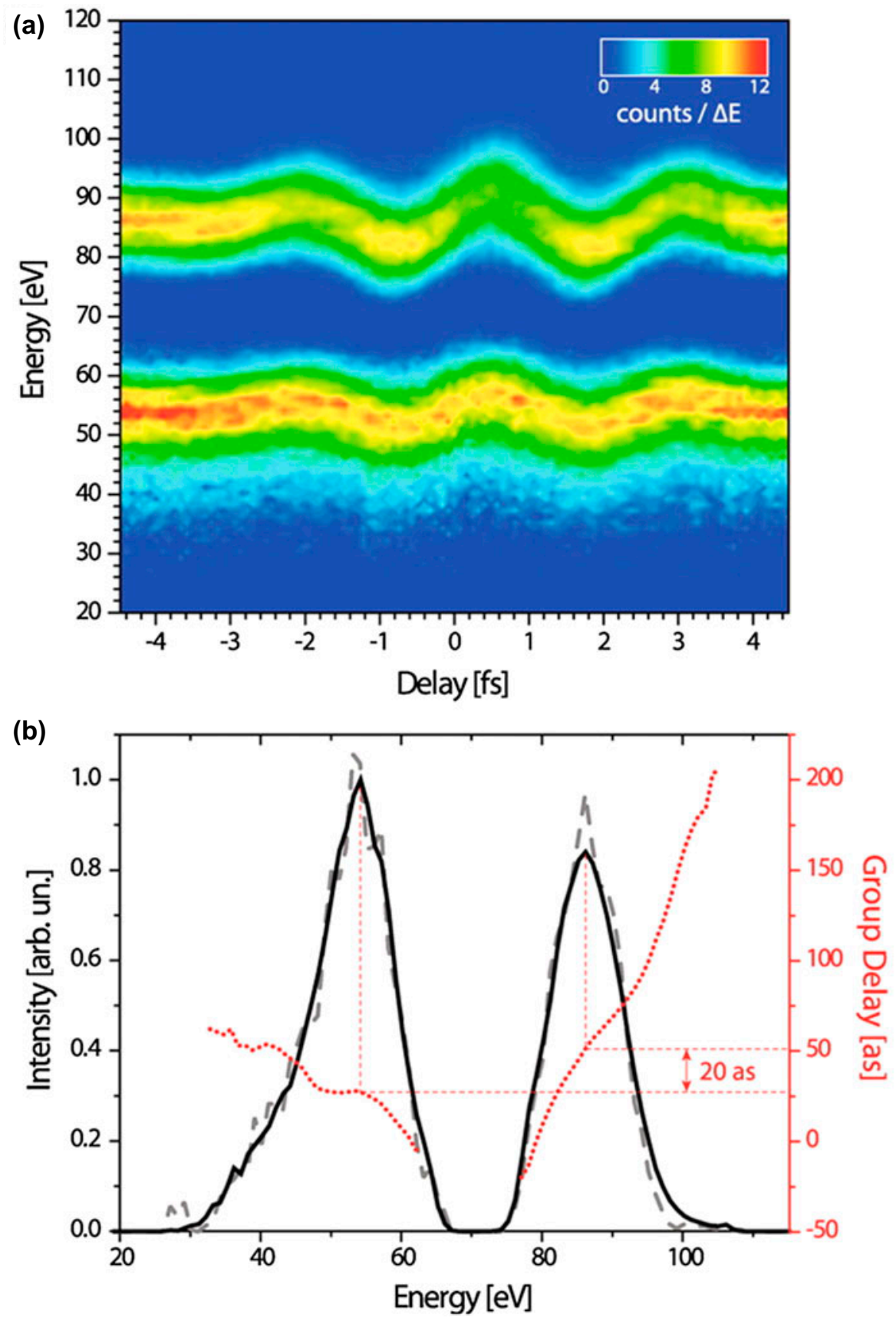

Figure 14. Delay in photoemission of the $2 s$ and $2 p$ shells of neon. (a) Photoelectron kinetic energy spectrum vs delay between infrared and attosecond pulses. The kinetic energy is modulated by the presence of the infrared dressing field. (b) Retrieved $2 \mathrm{~s}$ and $2 \mathrm{p}$ photoelectron spectra and group delays. The inferred 2s-2p time delay is 20 asec. (Figure is derived from Ref. [46] with permission of Nature Publishing Group.)

the Eisenbud-Wigner-Smith delay to give credit to its various originators. The time delay is written as

$$
\tau_{W}(\epsilon)=\hbar \frac{d \eta_{\ell}}{d \epsilon} .
$$

Here, $\ell$ labels the angular momentum of the final state of the transition.

To illustrate this effect, we look at the phase shift of the transition dipole moment for photoionisation of an atom having a centrally symmetric potential $V(r)$. In this case, Schrodinger's equation separates into a radial component and an angular component, giving a solution of the form

$$
\psi_{c}(r, \theta, \phi)=\sum_{\ell m} \frac{1}{r} R_{\ell}(r) Y_{\ell m}(\theta, \phi),
$$

where $R_{\ell}$ is the radial wavefunction and $Y_{\ell m}$ are spherical harmonics. $R$ depends on the exact form of the potential 
(a) Photon Energy (units of $\omega$ )

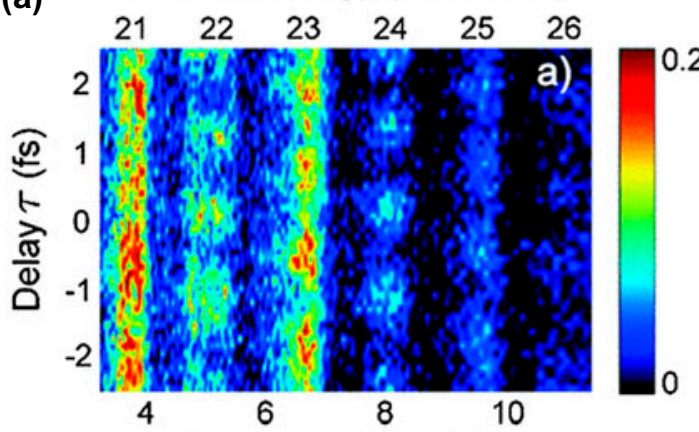

Kinetic Energy (eV) (b) Photon Energy (units of $\omega$ )

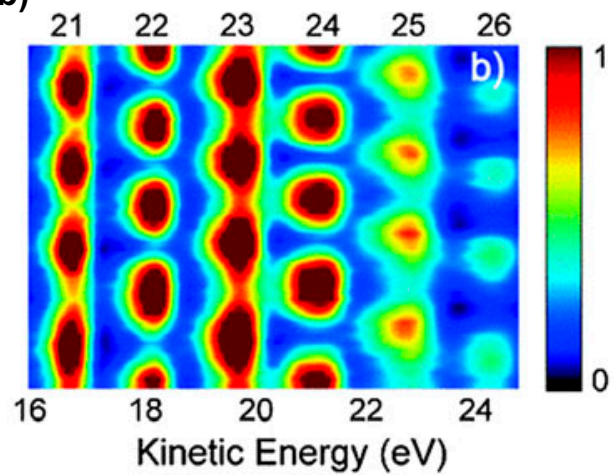

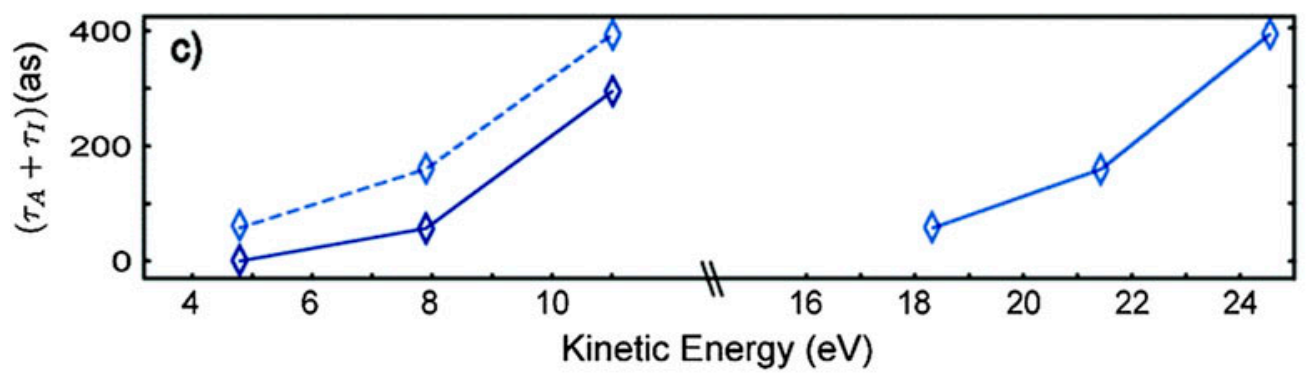

Figure 15. Photoionisation of argon atoms using an attosecond pulse train. (a) Photoelectron kinetic energy vs delay between infrared and XUV, for electrons originating from the $3 \mathrm{~s}$ shell. (b) Electrons from the $3 p$ shell. (c) Retrieved photoionisation delay times. (Figure is derived from Ref. [42] with permission of the American Physical Society.)

$V(r)$ as well as the photon energy, but the angular part is treated analytically using angular momentum algebra. The photon adds or subtracts one unit of angular momentum, so an initial $p$ state can result in a continuum wavefunction with $s$ or $d$ character.

The process to determine the phase shift (which is $\arg R$ ) is described by Starace [41]. Numerical solutions of the reduced Schrodinger equation for $R$ must match the asymptotic boundary condition which is the asymptotic form of the spherical Bessel function for a Coulomb potential [41,42],

$$
\lim _{r \rightarrow \infty} R_{k \ell}(r)=\sin \left(k r-\frac{\ell}{2} \pi+\frac{1}{k} \ln (2 k r)+\eta_{\ell}(k)\right) .
$$

The $\eta_{\ell}(k)$ is the desired phase shift, where $k$ is the final momentum of the outgoing electron given by the relation $\epsilon=I_{p}+\frac{1}{2} k^{2}$. Because photoionisation and scattering are time-reversed versions of one another, $\eta$ is called the scattering phase shift. Its derivative with respect to energy gives the Wigner time delay (Equation (11)). This atomic treatment can be generalised to the case of molecules [43].

A complication to the above picture emerges when an infrared laser field is used to streak the photoelectrons. The infrared field adds an additional delay term called the continuum-continuum delay that can be as large as the Wigner delay itself $[40,42,44]$. Furthermore, electronelectron correlations will change this single-electron picture [45]. The ability to observe such correlations, which are ignored in mean-field quantum chemistry calculations, is one of the goals of attosecond science.

Figure 14 shows an experiment which explored the time required to remove $2 s$ and $2 p$ electrons from neon atoms. Just as with the pulse characterisation methods described earlier, the infrared laser pulse that creates the attosecond pulse is used to streak the photoelectrons from neon. Isolated attosecond pulses with duration less than 100 asec are generated, with photon energies around $100 \mathrm{eV}$ selected by multilayer dielectric mirrors. The measurement shows that the $2 \mathrm{p}$ photoelectrons lag the 2 s photoelectrons by 20 asec. This delay is larger than expected [45].

A similar experiment was performed to compare the ionisation delay between $3 \mathrm{~s}$ and $3 \mathrm{p}$ electrons from argon [42]. Instead of using isolated attosecond pulses, an attosecond pulse train was used. The number of harmonic orders was limited by use of a chromium filter, in order to allow the photoelectrons from each orbital to be separated in kinetic energy. Figure 15 shows the experimental results. The photoelectron kinetic energy spectra exhibit sidebands due to the modulation of the infrared dressing field, as the RABBIT measurement in Equation (9). In the RABBIT measurement, it is the phase between harmonic orders that is sought. In the argon experiment, it is the atomic phase difference that is sought. By comparing the two different ionisation channels using the same attosecond pulse train, the harmonic phase cancels, leaving the atomic phase. This work also highlights 

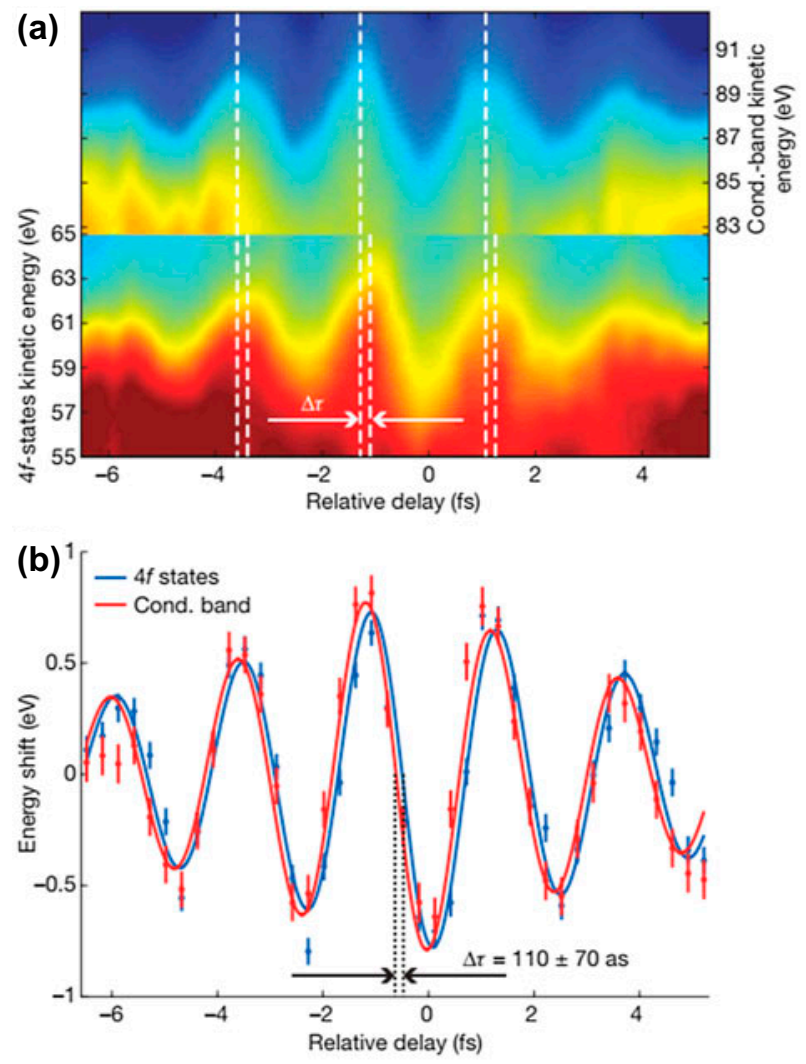

Figure 16. A single attosecond pulse removes electrons from a tungsten surface. A small delay between conduction band electrons and more deeply bound $4 \mathrm{f}$ electrons is seen. (a) Streaking spectra of electrons from the conduction band (top) and $4 \mathrm{f}$ (bottom). (b) Average energy shift of photoelectron kinetic energy as a function of time delay between the attosecond pulse and the infrared laser pulse, showing that the $4 f$ electron emerges 110 asec after the conduction band electron. (Figure is derived from Ref. [47] with permission of Nature Publishing Group.)

the fact that experiments do not directly measure the photoionisation delay. The additional effect of transitions in the continuum due to the infrared pulse must be included as discussed above and can actually change the sign of the relative delay. This work concluded that the $3 \mathrm{~s}$ photoelectron 'appears' before the $3 \mathrm{p}$ photoelectron.

Photoionisation delay measurements are not limited to atoms. A pioneering experiment measured the time delay to remove electrons from a tungsten surface [47]. This type of experiment is challenging due to the ultrahigh vacuum requirements to keep the surface free of oxides. An attosecond pulse was generated in a neon gas cell, producing a spectrum from 30 to $100 \mathrm{eV}$. When incident on the tungsten surface, it produced photoelectrons at 80 $\mathrm{eV}$ from the conduction band, as well as another peak at $55 \mathrm{eV}$ due to removal of more deeply bound electrons associated with the $4 \mathrm{f}$ state. An infrared laser pulse synchronised with the attosecond pulse provided a streaking

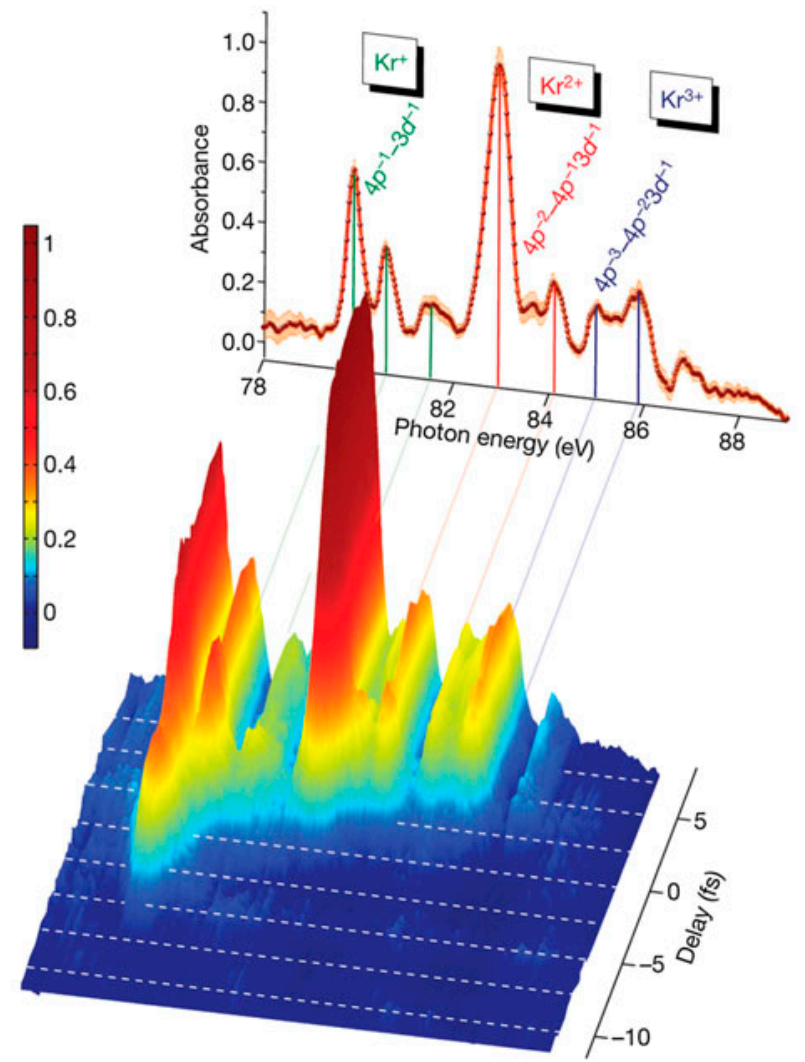

Figure 17. Transient absorption in ionised krypton. The lower figure shows the absorbance caused by transmission of an attosecond pulse propagating through a krypton gas cell as a function of photon energy and of time delay between the attosecond pulse and the infrared pulse that is ionising the krypton atoms. The upper figure shows the identification of the transitions associated with each absorption line. The delay of onset of the absorption lines is a measure of the delay in reaching that charge state of the ion. (Figure is derived from Ref. [49] with permission of Nature Publishing Group.)

field for the electrons. As seen in Figure 16, a slight shift in the photoelectron streaking pattern is associated with a 110 attosecond delay of the deeply bound $4 \mathrm{f}$ electrons relative to the valence electrons.

\section{Transient absorption}

In the previous sections, we showed how attosecond pulses can be used to measure fast processes in atoms, through photoionisation. The kinetic energy spectrum of the liberated electrons, in the presence of an infrared dressing field, is used to infer the temporal characteristics of the process.

Another attosecond measurement technique is called attosecond transient absorption. Transient absorption is the process in which a short-duration broadband pulse propagates through an absorbing medium. The absorption lines are impressed upon the transmitted spectrum and are caused by electronic transitions within the 


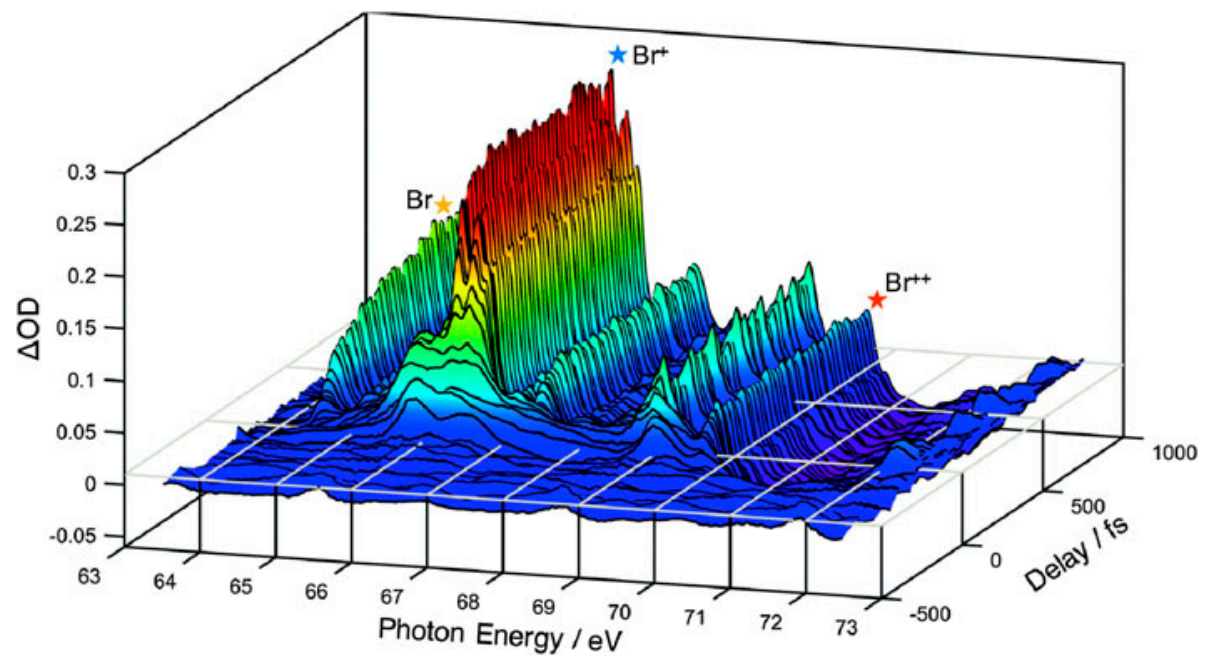

Figure 18. Transient absorption spectrum of dibromomethane as a function of pump-probe time delay. The pump pulse is an infrared pulse with an intensity of $1.5 \times 10^{14} \mathrm{~W} / \mathrm{cm}^{2}$ and serves to ionise and then dissociate the molecule. The transient absorption probe records the fragments and their charge states. (Reproduced from Ref. [51] with permission from the PCCP Owner Societies.)

medium, just as regular absorption of an incoherent light source. At first, it seems paradoxical that the narrow absorption lines appear on the broad spectrum for the short pulse, seemingly in violation of the Fourier transform limit. Of course there is no violation. Instead, the short pulse creates an electronic coherence between electronic states. This electronic coherence is associated with an oscillating dipole moment that radiates for picoseconds or even nanoseconds at a frequency given by the energy difference between states. The long emission time then supports a narrow absorption feature. The emission must also be $\pi$ out of phase with the excitation pulse.

An impressive demonstration of the ability to create and modify the coherences was shown by Ott et al. [48] who used an attosecond pulse to ionise helium atoms. Around $64 \mathrm{eV}$, there is a doubly excited state which forms absorption lines with a Fano profile. By applying an infrared pulse after the attosecond pulse, the line shape could be changed to a Lorentzian profile. Similarly, singly excited states just below the ionisation limit at $25 \mathrm{eV}$ were produced with Lorentzian line profiles, that were changed to Fano profiles by applying the infrared pulse.

Another attosecond transient absorption experiment probed the ionisation state of krypton atoms [49]. An intense few-cycle infrared laser pulse ionised a krypton gas sample. An isolated attosecond pulse then passed through the gas sample. The spectrum, in the range 76$96 \mathrm{eV}$, showed absorption lines due to $4 \mathrm{p}-3 \mathrm{~d}$ bound transitions. The absorption as a function of time delay is shown in Figure 17. Since the $4 \mathrm{p}-3 \mathrm{~d}$ transition frequency moves higher as the charge state of the atom increases, the charge state can be measured as a function of time. It can be seen that the $\mathrm{Kr}^{+3}$ occurs later than the $\mathrm{Kr}^{+1}$ state. The experiment was able to measure the degree of electronic coherence of the ionic states populated by the infrared laser, which is different from the case of holes produced by attosecond pulses. A density matrix approach is needed to describe the hole states produced by the infrared laser [50]. A very short infrared pulse is needed to produce a high degree of coherence of the hole state.

Attosecond transient absorption spectroscopy was used to track the dissociation dynamics of the molecule dibromoethane [51]. The $800-\mathrm{nm}$ laser pulse is used as a pump pulse to excite and ionise the molecules. The same pulse created a continuous XUV spectrum covering 50 to $73 \mathrm{eV}$ that served as the probe pulse. As for krypton, the absorption lines shift to higher energy with increasing charge state of the molecule. Figure 18 shows the measured absorption spectrum as a function of pump-probe time delay. The peak due to the parent bromine atom disappears around time zero and peaks due to excited bromine appear at later times. From this picture, it was possible to infer time constants associated with the dissociation of the parent molecule. This work demonstrates the promise of attosecond transient absorption spectroscopy to study molecular dynamics.

\section{Conclusions}

Laboratory sources of attosecond pulses have only been available since 2001. The shortest such pulse recorded so far has a duration of 53 asec [2]. Initial work in this field concentrated on generating and characterising the attosecond pulses. Then attosecond pulses were used to study fundamental physical processes such as the time 
delay to remove an electron from an atom or from a surface. More recently the spectral continuum associated with attosecond pulses has been used as a probe in a transient absorption setting, to observe holes and excitations in atoms and molecules. Single attosecond pulses whose spectrum spans the carbon K-edge around $280 \mathrm{eV}$ are now available to apply near-edge X-ray absorption structural measurement techniques with attosecond resolution. We expect that further applications of attosecond technology will continue to emerge. The interested reader is directed to related review articles [52-54].

\section{Disclosure statement}

No potential conflict of interest was reported by the author.

\section{Notes on contributor}

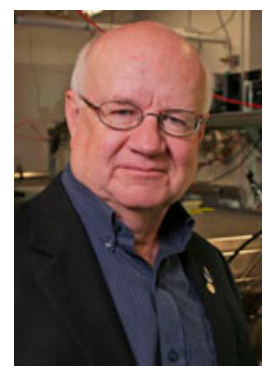

D. M. Villeneuve is currently the head of the attosecond science group at the National Research Council of Canada, and part of the Joint Attosecond Science Laboratory between the National Research Council and the University of Ottawa. He is a Fellow of the Royal Society of Canada, of the American Physical Society, and of the Optical Society of America.

\section{ORCID}

D. M. Villeneuve (iD http://orcid.org/0000-0002-2810-3648

\section{References}

[1] Edgerton HE. Electronic flash, strobe. 3rd ed. Cambridge (MA): The MIT Press; 1987.

[2] Li J, Ren X, Yin Y, et al. 53-attosecond X-ray pulses reach the carbon K-edge. Nat Commun. 2017;8:186.

[3] Milonni PW, Eberly JH. Laser physics. Hoboken (NJ): Wiley; 2009.

[4] Reagan BA, Berrill M, Wernsing KA, et al. High-averagepower, $100-\mathrm{Hz}$-repetition-rate, tabletop soft-x-ray lasers at sub-15-nm wavelengths. Phys Rev A. 2014;89:053820.

[5] McPherson A, Gibson G, Jara H, et al. Studies of multiphoton production of vacuum-ultraviolet radiation in the rare gases. JOSA B. 1987;4:595-601.

[6] Ferray M, L'Huillier A, Li XF, et al. Multiple-harmonic conversion of $1064 \mathrm{~nm}$ radiation in rare gases. J Phys $\mathrm{B}$. 1988;21:L31-L35.

[7] Wahlström C-G, Larsson J, Persson A, et al. High-order harmonic generation in rare gases with an intense shortpulse laser. Phys Rev A. 1993;48:4709-4720.

[8] Krause JL, Schafer KJ, Kulander KC. High-order harmonic generation from atoms and ions in the high intensity regime. Phys Rev Lett. 1992;68:3535-3538.

[9] Corkum PB, Burnett NH, Ivanov MY. Subfemtosecond pulses. Opt Lett. 1994;19:1870-1872.
[10] Corkum PB. Plasma perspective on strong field multiphoton ionization. Phys Rev Lett. 1993;71:19941997.

[11] Lewenstein M, Balcou P, Ivanov MY, et al. Theory of highharmonic generation by low-frequency laser fields. Phys Rev A. 1994;49:2117-2132.

[12] Burnett K, Reed VC, Cooper J, et al. Calculation of the background emitted during high-harmonic generation. Phys Rev A. 1992;45:3347-3349.

[13] Itatani J, Levesque J, Zeidler D, et al. Tomographic imaging of molecular orbitals. Nature. 2004;432:867-871.

[14] Gilbertson S, Mashiko H, Li C, et al. A low-loss, robust setup for double optical gating of high harmonic generation. Appl Phys Lett. 2008;92:071109.

[15] Abel M, Pfeifer T, Nagel P, et al. Isolated attosecond pulses from ionization gating of high-harmonic emission. Chem Phys. 2009;366:9-14.

[16] Kim KT, Zhang C, Ruchon T, et al. Photonic streaking of attosecond pulse trains. Nat Photon. 2013;7:651-656.

[17] Sansone G, Benedetti E, Calegari F, et al. Isolated singlecycle attosecond pulses. Science. 2006;314:443-446.

[18] Shan B, Chang Z. Dramatic extension of the high-order harmonic cutoff by using a long-wavelength driving field. Phys Rev A. 2001;65:011804.

[19] Manzoni C, Cerullo G. Design criteria for ultrafast optical parametric amplifiers. J Opt. 2016;18:103501.

[20] Witte S, Eikema KSE. Ultrafast optical parametric chirped-pulse amplification. IEEE J Sel Top Quantum Electron. 2012;18:296-307.

[21] Schmidt BE, Thiré N, Boivin M, et al. Frequency domain optical parametric amplification. Nat Commun. 2014;5:3643. DOI:10.1038/ncomms4643

[22] Tate J, Auguste T, Muller HG, et al. Scaling of wave-packet dynamics in an intense midinfrared field. Phys Rev Lett. 2007;98:013901.

[23] Shiner AD, Trallero-Herrero C, Kajumba N, et al. Wavelength scaling of high harmonic generation efficiency. Phys Rev Lett. 2009;103:073902.

[24] Yakovlev VS, Ivanov M, Krausz F. Enhanced phasematching for generation of soft X-ray harmonics and attosecond pulses in atomic gases. Opt Express. 2007;15:15351-15364.

[25] Popmintchev T, Chen M-C, Popmintchev D, et al. Bright coherent ultrahigh harmonics in the $\mathrm{keV} \mathrm{X}$-ray regime from mid-infrared femtosecond lasers. Science. 2012;336:1287-1291.

[26] Balcou P, Salières P, L'Huillier A, et al. Generalized phasematching conditions for high harmonics: the role of fieldgradient forces. Phys Rev A. 1997;55:3204-3210.

[27] Salières P, L'Huillier A, Lewenstein M. Coherence control of high-order harmonics. Phys Rev Lett. 1995;74:37763779.

[28] Kim KT, Villeneuve DM, Corkum PB. Manipulating quantum paths for novel attosecond measurement methods. Nat Photon. 2014;8:187-194.

[29] Paul PM, Toma ES, Breger P, et al. Observation of a train of attosecond pulses from high harmonic generation. Science. 2001;292:1689-1692.

[30] Mairesse Y, de Bohan A, Frasinski LJ, et al. Attosecond synchronization of high-harmonic soft X-rays. Science. 2003;302:1540-1543. 
[31] Kim KT, Kim CM, Baik M-G, et al. Single 50 attosecond pulse generation from chirp-compensated harmonic radiation using material dispersion. Phys Rev A. 2004;69:051805.

[32] Itatani J, Quéré F, Yudin GL, et al. Attosecond streak camera. Phys Rev Lett. 2002;88:173903-173903.

[33] Mairesse Y, Quéré F. Frequency-resolved optical gating for complete reconstruction of attosecond bursts. Phys Rev A. 2005;71:011401.

[34] Spanner M, Bertrand JB, Villeneuve DM. In situ attosecond pulse characterization techniques to measure the electromagnetic phase. Phys Rev A. 2016;94:023825.

[35] Dudovich N, Smirnova O, Levesque J, et al. Measuring and controlling the birth of attosecond XUV pulses. Nat Phys. 2006;2:781-786.

[36] Kim KT, Zhang C, Shiner AD, et al. Petahertz optical oscilloscope. Nat Photon. 2013;7:958-962.

[37] Kim KT, Zhang C, Shiner AD, et al. Manipulation of quantum paths for space-time characterization of attosecond pulses. Nat Phys. 2013;9:159-163.

[38] Vincenti H, Quéré F. Attosecond lighthouses: how to use spatiotemporally coupled light fields to generate isolated attosecond pulses. Phys Rev Lett. 2012;108:113904.

[39] Hammond TJ, Brown GG, Kim KT, et al. Attosecond pulses measured from the attosecond lighthouse. Nat Photon. 2016;10:171-175.

[40] Pazourek R, Nagele S, Burgdörfer J. Attosecond chronoscopy of photoemission. Rev Mod Phys. 2015;87:765-802.

[41] Starace AF. Theory of atomic photoionization. In: Mehlhorn W, editor. Handbuch Der Physik. Vol. XXXI. Berlin: Springer; 1982. p. 1-121.

[42] Klünder K, Dahlström J, Gisselbrecht M, et al. Probing single-photon ionization on the attosecond time scale. Phys Rev Lett. 2011;106:143002.
[43] Hockett P, Frumker E, Villeneuve DM, et al. Time delay in molecular photoionization. J Phys B At Mol Opt Phys. 2016;49:095602.

[44] Dahlström JM, Guénot D, Klünder K, et al. Theory of attosecond delays in laser-assisted photoionization. Chem Phys. 2013;414:53-64.

[45] Kheifets A, Ivanov I. Delay in atomic photoionization. Phys Rev Lett. 2010;105:233002.

[46] Schultze M, Fieß M, Karpowicz N, et al. Delay in photoemission. Science. 2010;328:1658-1662.

[47] Cavalieri A, Muller N, Uphues T, et al. Attosecond spectroscopy in condensed matter. Nature. 2007;449:10291032.

[48] Ott C, Kaldun A, Raith P, et al. Lorentz meets Fano in spectral line shapes: a universal phase and its laser control. Science. 2013;340:716-720.

[49] Goulielmakis E, Loh Z-H, Wirth A, et al. Realtime observation of valence electron motion. Nature. 2010;466:739-743.

[50] Goetz RE, Merkel M, Karamatskou A, et al. Maximizing hole coherence in ultrafast photoionization of argon with an optimization by sequential parametrization update. Phys Rev A. 2016;94:023420.

[51] Chatterley AS, Lackner F, Neumark DM, et al. Tracking dissociation dynamics of strong-field ionized 1,2-dibromoethane with femtosecond XUV transient absorption spectroscopy. Phys Chem Chem Phys. 2016;18:14644-14653.

[52] Scrinzi A, Ivanov MY, Kienberger R, et al. Attosecond physics. J Phys B At Mol Opt Phys. 2006;39:R1-R37.

[53] Corkum PB, Krausz F. Attosecond science. Nat Phys. 2007;3:381-387.

[54] Krausz F, Ivanov M. Attosecond physics. Rev Mod Phys. 2009;81:163-234. 